\title{
Tangence
}

\section{Lire, comprendre, interpréter}

\section{Gilles Thérien}

Numéro 36, mai 1992

La lecture littéraire

URI : https://id.erudit.org/iderudit/025715ar

DOI : https://doi.org/10.7202/025715ar

Aller au sommaire du numéro

Éditeur(s)

Tangence

ISSN

0226-9554 (imprimé)

1710-0305 (numérique)

Découvrir la revue

Citer cet article

Thérien, G. (1992). Lire, comprendre, interpréter. Tangence, (36), 96-104.

https://doi.org/10.7202/025715ar d'utilisation que vous pouvez consulter en ligne.

https://apropos.erudit.org/fr/usagers/politique-dutilisation/ 


\section{Lire, comprendre, interpréter}

\section{Gilles Thérien}

Le déploiement simultané des trois verbes «lire", "comprendre" et "interpréter" ne peut paraître qu'une forme de mise en place linéaire de description de la lecture. Est-ce qu'en effet "lire" ne suppose pas toujours "comprendre" et "interpréter "? Nous pourrions facilement nous mettre d'accord sur pareille forme de truisme qui reproduit de façon globale les processus d'apprentissage de la lecture et de sa pratique, et le tout serait dit. Si j'ai voulu reprendre ici les trois termes côte à côte, c'est pourtant justement parce que cela ne me semblait pas aller de soi, se faire sans heurts et donner les résultats escomptés de la façon la plus parfaite possible. Au fond, nous ne faisons pas que suivre le mode d'emploi. Le titre n'est pas "lire: comprendre et interpréter ". Les trois actions ne sont pas de même nature et n'entraînent pas les mêmes réactions de la part du lecteur. C'est là-dessus que je veux insister. Lire n'est pas une activité simple, pas plus qu'une simple activité.

Avant de passer en revue les trois éléments de cette problématique, il me semble utile de répéter ici et encore que je me place dans une perspective de lecture littéraire et que cette lecture, dont j'ai parlé ailleurs ${ }^{1}$, se fait par l'intermédiaire d'un acte de lecture complexe, privé, intime et singulier et qu'il ne sert à rien de réfléchir et d'épiloguer sur la lecture si l'on ne tient pas compte de ces divers aspects de sa performance.

\section{Lire}

Dès la toute première évocation du "lire", il faut comprendre le bond que l'on vient d'accomplir. Le "lire", dı domaine de l'écrit, de la graphie, s'oppose à l'oral. Il n'en est pas la continuation normale, la mise en mots d'une parole sonore, le complément par lequel l'oral devient de l'écrit. En ce sens et du même

1 Voir "Pour une sémiotique de la lecture», Protée, vol. XVIII, no 2, p. 6780 . 
coup, je peux affirmer que littérature orale et littérature écrite n'appartiennent pas au même monde, au même univers mental. D'ailleurs, on ne devrait pas dire "littérature orale". On l'a reconnu sur le plan scientifique en confiant la tradition orale aux folkloristes et la littérature aux littéraires. L'écrit littéraire entraîne non seulement l'usage de signes graphiques bien précis qui n'ont aucun rapport avec le monde naturel mais il comprend, comme condition de son propre accomplissement, le travail des mots et sur les mots, la maîtrise des phrases selon des unités de diverses longueurs comme il suppose la maitrise du rythme visuel comme partie co-fondatrice de la cohérence du discours littéraire. En somme, il ne suffit pas dans l'acte de lecture littéraire de savoir décoder les mots que l'on retrouve à la fois dans le dictionnaire, dans le journal et dans la conversation quotidienne, mais il s'agit de comprendre que ces mots, arrachés au domaine de l'oralité, sont du même coup arrachés à l'emprise de la communication. La lecture littéraire ne s'établit pas sur le mode de l'échange, fantômes d'auteurs qui hantent les imaginaires de lecteurs, mais elle s'accomplit dans une perspective de décodage intime, personnel, privé, d'un objet fait de mots que l'acte de lecture entreprend de constituer, de construire.

La toute première activité du "lire", c'est donc le décodage de la langue littéraire et non de la langue commune de tous les jours. C'est la prise en compte des pièges de la graphie, de l'orthographie, des accords. C'est la reconnaissance des segmentations, des espaces blancs qui distribuent sur la page l'objet littéraire selon des contours bien précis. On peut ici, à titre d'exemple, opposer la page écrite sans interruption d'un Thomas Bernhard à celle toute morcelée de Beckett. Ce sont ces signes, et plusieurs autres, qui s'ajoutent à l'usage ordinaire de la langue, qui l'éloignent de la parole, de la communication dont les encodages-décodages sont simplifiés et vérifiables à l'intérieur même de l'acte de communication. Lire le littéraire, c'est faire état d'une double performance de lecture, une performance qui s'appuie sur la connaissance de la langue et l'autre sur la connaissance et la pratique de la lecture littéraire. On peut connaître une langue assez imparfaitement et être capable de comprendre en gros ce qu'elle veut dire. C'était le cas, pendant des générations, du latin d'Église au Québec. C'est aussi le cas d'une langue imparfaitement maîtrisée que l'on commence à apprendre, l'allemand, l'italien, l'espagnol, langues que l'on peut "lire" à voix haute avec une grande précision, mais 
dont la connaissance insuffisante ne nous permet pas de nous faire une idée de ce dont il est question. Il faut plus que cela pour rendre une lecture littéraire possible ou valable.

Dans le "lire ", nous retrouvons à l'œuvre tous les processus de lecture ${ }^{2}$, mais il faut bien noter, une fois de plus, que ces processsus sont ici, déjà, dans la sphère du littéraire. Il ne s'agit pas seulement de percevoir des mots, mais d'être capable de percevoir la mise en scène graphique d'un roman, d'un poème, du paratexte, des jeux de langues qui renvoient parfois le lecteur à sa propre incompétence, tels ces textes en hébreu cités dans le dernier roman d'Eco, Le pendule de Foucault. De même, le processus affectif ne peut ici se limiter à une sorte de curiosité bienveillante ou d'agressivité malveillante à l'endroit d'un journal ou d'un texte aussi peu littéraire qu'un compte à payer ou une contravention. La lecture littéraire engage les affects qui deviennent indispensables à la lecture même car leur intensité va permettre au lecteur de créer des noyaux, des topoi mémoriels qui l'aideront à structurer sa démarche à travers le roman. Il n'en va pas autrement du processus cognitif qui ne peut être rabattu sur une mimésis du vécu sans risquer de faire disparaître une partie importante de la littérature, et non la moindre, la poésie et tous ces textes de la modernité qui refusent justement le piège si bien tendu par le succès du roman réaliste. La cognition appliquée au domaine littéraire ne peut être la cognition en général, mais la saisie d'un univers cognitif qui s'est constitué par diverses pratiques littéraires souvent hétérogènes les unes par rapport aux autres, mais reconnues par l'institution littéraire, comme les glossolalies de Gauvreau ou d'Artaud. Cet univers cognitif spécifique repose fondamentalement sur des mots et des discours. Il résiste à toute confrontation avec une référence objectale, même si l'on peut tracer un parallèle entre tel objet et telle phrase. Le processus argumentatif suppose, ici, l'acquisition d'une connaissance progressive du domaine de la littérature et de ses techniques. L'argumentation, qui peut prendre la forme simple du récit linéaire ou celle, plus difficile, du labyrinthe, doit aussi tenir compte des figures qui marquent son parcours. En littérature, aucun mot n'est un mot du dictionnaire, aucune phrase n'est une phrase de la vie quotidienne. Les transformations rhétoriques sont responsables de la métamorphose de la langue ordinaire en langue littéraire. On

Ibid. 
n'a pour s'en convaincre qu'à réfléchir au sens de l'expression "parler comme un grand livre". Le processus symbolique est un processus intégrateur qui engrange les lectures, les fait converger, les met en écho. Ici, dans le "lire", il intègre tout autant le contenu de la lecture que les éléments de son expérience. Ces éléments sont d'un niveau parfois très personnel, redevables de sa seule perspective, de son expérience unique. Parfois, ils sont d'un ordre plus technique comme, lorsqu'en lisant, je fais aussi l'expérience d'une nouvelle réalité littéraire, de la façon originale de créer un nouvel objet littéraire ou encore d'un savoir qui me manquait. Mais l'intégration, c'est aussi la sélection plus ou moins consciente d'éléments qui transitent dans mon imaginaire soit comme totalité, soit comme fragments, pour conforter les idées reçues qui constituent cet imaginaire personnel, pour les ébranler et, dans quelques cas rares, pour ouvrir l'imaginaire à de nouvelles dimensions. La lecture littéraire est un processus virtuel d'enrichissement du champ symbolique personnel.

"Lire ", dans le domaine de la lecture littéraire, est déjà un acte très complexe que je ne peux réduire à la répétition mentale de mots ou de phrases. La signification est déjà engagée de même que le sens mais la lecture est un processus de durée qui empêche normalement de tout réduire à quelques opérations de décodage qui auront tôt fait de rendre compte d'une lecture tout en la vidant de son contenu "expérienciel".

\section{Comprendre}

Dans le cadre de réflexion que je propose, il est évident que "comprendre" ne peut se substituer à lire. Il n'est pas une activité dont on peut faire l'économie. "Comprendre" dépasse le simple niveau de la lecture parce qu'il engage à la fois les tentatives de comparaison et les activités intellectuelles de catégorisation.

La façon dont je pense le "comprendre" suppose une double direction. La première a trait à la construction mentale de l'objet littéraire, la seconde prend en compte les diverses catégories conceptuelles qui permettent de réaliser le "comprendre".

"Comprendre" une œuvre littéraire, ce n'est certainement pas résoudre une énigme. C'est, au sens étymologique du terme, "prendre-avec-soi " les divers fils qui se nouent et se dénouent au cours d'une œuvre, les suivre pour arriver à créer un objet mental 
qui soit le résultat de notre lecture de tel ou tel livre. Dans cet effort de compréhension, il faut tout de suite accepter que l'exhaustivité est impossible et qu'il est également impossible de poser une équivalence absolue entre l'objet construit par le lecteur et le livre qui lui a servi de point de départ et d'accompagnement dans son expérience. Comprendre, c'est construire. C'est donc pouvoir, en cours de lecture, poser les jalons de la construction de l'objet littéraire, c'est-à-dire sa cohésion et sa cohérence. Cela suppose une gestion des éléments qui, par la sérialisation des micro-structures, entraîne la mise en place progressive de la macro-structure de tel ou tel texte, en même temps que le lecteur est constamment appelé, dans l'ordre de la compréhension, à contrôler la construction de l'objet mental, résultat de sa lecture, faute de quoi il devra abandonner telle ou telle lecture parce qu'elle ne pourra plus servir à la construction de son propre objet. Comme le tout se passe dans la durée - pensons aux ouvres les plus longues comme aux plus courtes -, c'est la mémoire qui assume la compréhension. Mais, ici, il faut faire grandement attention au mot "mémoire". Il ne s'agit pas de cette faculté que l'on retrouve habituellement dans les manuels de psychologie ou encore dans des expressions comme "avoir une mémoire d'éléphant "ou "je perds la mémoire". La mémoire, dont il est ici question, est en fait la memoria de la rhétorique ancienne qu'il est urgent de ramener au centre de la réflexion littéraire 3 . "Comprendre ", ce n'est pas se souvenir et organiser ce qu'on a déjà lu, comme s'il était possible de jouer avec les résidus d'un passé plus ou moins lointain qui auraient gardé une sorte d'authenticitê ou de pureté qui leur assurerait une transparence au niveau de leur usage et de leur organisation. "Comprendre", c'est construire une memoria spécifique qui correspond progressivement et, de mieux en mieux, à l'objet littéraire construit dans l'acte de lecture. La memoria, c'est la mise en scène mentale de l'objet littéraire. C'est là, et non sur la page écrite, que les personnages se rencontrent, qu'ils interviennent et qu'ils poursuivent une destinée de papier que la memoria justifie constamment en se déployant en même temps que leur va-et-vient.

Cette mémoire nous oblige à retenir des catégories qui vont se retrouver dans le plus grand nombre de ces constructions,

3 Voir, entre autres, Mary Carruthers, The Book of Memory, New York/ Cambridge, Cambridge University Press, 1990. 
sinon dans toutes. Ce sont les préconstruits, c'est-à-dire l'espace, le personnage, l'action et le temps. Ces préconstruits peuvent avoir un petit air kantien en ce sens qu'ils sont posés a priori par rapport à la lecture littéraire. Pourtant, s'ils sont posés a priori, ce n'est pas au niveau de catégories vides et essentielles dont il faut toujours tenir compte, mais plutôt au niveau d'un réseau d'homologies entre le sujet humain, la langue et l'objet littéraire, du moins celui qui se définit par le romanesque. Sur le plan du sujet humain, espace, sujet, action et temps sont des constantes de l'expérience. L'espace, c'est d'abord l'image de son corps propre et, aussi, le territoire dans lequel le sujet apprend à se mouvoir. Le sujet, c'est évidemment la personne en tant qu'elle peut dire "je" et s'attribuer des pensées, des intentions, une volonté. J'évite ici le mot "conscience" qui me semble déjà trop chargé de ces divers éléments qui constituent une identité, une idéologie, un imaginaire personnel. Il suffit, dirait Occam, d'un sujet à intentionalité minimale, capable de se reconnaître dans un "je", mais qui n'a pas besoin pour se définir de la somme de toutes ses expériences. Le mot "action" exige moins d'explications et de précautions. Il s'agit des actions proprement dites que toute personne accomplit, que ces actions en soient de véritables ou qu'il s'agisse d'états entre diverses actions, états qui sont toujours plus ou moins passagers, provisoires. Enfin, le temps est une variable qui permet au sujet de poser dans un espace plus ou moins défini diverses actions, divers lieux et de les relier dans un ordre chronologique. Le temps est alors non pas une donnée ferme, fixe, mais la description des étapes d'une démarche. Ces préconstruits se retrouvent dans la langue, d'où le second rapport homologique. La personne devient cette fois le sujet, pronom ou nom, les actions sont les verbes. Les lieux sont identifiés comme tels ou indiqués par la voie des déictiques et le temps fait à la fois partie de la construction du verbe, des déictiques et de la reconstitution chronologique entre les actions et les divers lieux. Il ne s'agit donc pas d'une mimésis entre ces deux niveaux, mais de catégories homologues qui permettent de passer d'un niveau à l'autre sans se perdre. Enfin, le troisième niveau d'homologies, celui que je pose de façon particulièrement insistante, se trouve dans l'objet littéraire et l'acte de lecture s'en sert pour établir une continuité entre le lecteur, ce qu'il lit et la langue qui lui sert de véhicule. La memoria, support de ces homologies, constitue la mise en scène de l'objet littéraire parce qu'elle est le lieu mental où ces diverses homologies viennent se confondre pour ne former que le résidu 
que chaque lecteur gardera, résidu formé alors autant de ce qu'il trouve dans le texte que de ce qu'il peut décoder de la langue ou investir de soi-même.

Dans le développement des études littéraires, diverses grilles mises au point aux plus beaux jours du structuralisme me semblent être une façon d'assurer plus statistiquement que scientifiquement une constance dans la lecture... chaque fois au prix évidemment de la position du lecteur parce que ce qui a toujours été implicite et tu dans ces grilles, c'est que tous les lecteurs passés et à venir étaient subsumés dans la position du seul lecteur architecte de la grille. La position que je défends ici sur le plan de la compréhension est loin de la mise en place d'une nouvelle grille. Elle a pour premier effet de redonner à chaque lecteur sa liberté face au texte en même temps qu'elle le rend responsable du plein travail de construction de l'objet littéraire mental. Chaque lecteur est responsable de sa lecture, chaque lecteur crée sa propre théorie. Cela ne veut aucunement dire que lectures et théories se multiplieront à l'infini. Loin de là! Le jeu des homologations aura toujours un point commun, un noyau dur, celui du texte littéraire à lire et à comprendre. Il ne s'agit pas d'une porte ouverte sur la polysémie ou le solipcisme, mais de la reconnaissance de l'homogénéité des éléments qui, au contraire, auront tendance à s'instaurer dans le cadre d'une "plurisêmie " produite et contrôlée par l'acte de lecture.

\section{Interpréter}

On pourrait croire que "lire" et "comprendre" suffisent à expliquer l'acte de lecture. "Interpréter" n'apparaît-il pas comme un terme chargé de sens multiples qu'il est difficile d'utiliser ici? Pourtant, il me semble que sa nécessité ne fait pas de doutes à la condition justement d'en préciser le sens et les enjeux.

"Lire " et "comprendre" sont des activités qui servent à construire mentalement l'objet littéraire. Dans un jeu de renvois avec le texte, il est même possible de fixer les diverses étapes de cette construction ou ses caractéristiques principales. Il est possible de les reproduire, de les enrichir. Alors, que peut bien ajouter l'interprétation à ces activités? Si l'interprétation est comprise comme le dévoilement d'un sens caché du texte, il est assez évident que cette activité est déjà comprise dans les deux précédentes. Un texte n'a pas de sens caché une fois qu'il est lu, il peut avoir d'autres sens que d'autres lectures mettront en lumière. 
En fait, l'interprétation du texte littéraire ressemble beaucoup à l'interprétation musicale. Quand un pianiste joue, par exemple, une pièce une première fois, partition devant lui, on ne pense pas nécessairement qu'il s'agisse d'une interprétation. On s'entendra pour parler d'une lecture et de la mise en place des différents éléments de compréhension. Quand le même pianiste se produit en concert et joue la même pièce, il s'agit alors à coup sûr d'une interprétation. Quelle est la différence entre ces deux moments? Dans la première phase, qui équivaut à notre "lire" et "comprendre", le pianiste s'approprie la pièce musicale. Il en découvre les traits principaux, il la reconstruit mentalement selon les éléments de compréhension qu'il a mis en place. Quand il interprète, cette pièce est assimilée. Elle existe déjà pour lui dans une antériorité et elle est sujette à diverses modifications selon les besoins ou même les impulsions du moment. Il peut lui donner un sens, du sens, son sens. La pièce fait maintenant partie de sa vie, de ses émotions, de sa pratique gestuelle. Il a acquis un droit de l'utiliser selon le sens qu'il veut lui insuffler. À tel moment, elle marquera un départ, une séparation, à tel autre, elle sera au contraire un moment de méditation... et chaque fois la reconstitution musicale matérielle de l'objet sera modifiée. C'est le sens que nous réservons ici au mot * interpréter ".

L'interprétation est d'abord une activité d'intégration qui se produit dans la durée. Elle permet au lecteur un retour actif sur son acte de lecture, une fois qu'il sait de quoi il est question. Les enjeux ne sont plus les mêmes. Il ne s'agit pas de trouver un sens à l'objet littéraire, mais bien d'y intégrer un sens pour soi. Le lecteur a le pouvoir de prendre ses lectures et de les assimiler de façon plus large dans sa culture personnelle, dans la perception qu'il a de la culture de son époque. Il peut sentir que tel objet l'exprime mieux que s'il avait cherché lui-même à s'exprimer sur le même sujet. Il peut prendre cet objet littéraire lu dans un contexte bien particulier et, dans sa memoria personnelle, lui assigner une nouvelle place, un nouvel environnement où, qu'il le veuille ou non, un nouveau sens apparaîtra. Mais ce sens ne peut être attribué à l'objet littéraire. Il est en fait le sens de la relation que l'objet littéraire entretient dans l'imaginaire du lecteur avec divers autres objets, diverses situations pas nécessairement littéraires mais qui forment son champ symbolique. Interpréter, c'est rendre parlante la place qu'occupe l'objet dans la symbolique du lecteur. Cette interprétation est unique. Elle peut être expliquée, comprise, mais elle demeure singulière et privée. 
"Interpréter", dans ce sens, c'est recourir à la richesse de l'analogie. Cette notion a connu bien des déveines. Pourtant, elle nous fournit ici une façon de montrer comment le processus d'interprêtation tisse des liens avec d'autres réalités au nom des similarités perçues. En même temps, puisqu'il s'agit bien d'analogie et non d'identité ou d'homologation, la différence apparaît comme la frontière contre laquelle vient buter le processus analogique. C'est dire que, dans le champ symbolique du lecteur, toute cuvre construite trouve un sens, une interprétation par le dosage réciproque des ressemblances et des différences. La frontière même entre ces deux états de choses est l'entière responsabilité du lecteur qui indique alors la manière dont il structure une partie de son champ symbolique. Interpréter, c'est établir des relations.

En conclusion, c'est dire que, dans le domaine de l'enseignement de la littérature, il est paradoxal de vouloir enseigner des interprétations comme résultats objectifs ou encore de faire de l'interprétation d'un texte en particulier une sorte de paradigme de la vérité. Ce que nous pouvons faire de mieux, c'est d'apprendre à distinguer deux phases pédagogiques, celle où il faut lire et comprendre, phase qui exige du travail, de l'attention, du temps, phase aussi qui repose largement sur les savoirs de l'institution littéraire, et une seconde phase, où il est simplement utile de montrer le processus par lequel l'interprétation peut se réaliser. Il entre d'ailleurs dans cette phase des considérations de sensibilité individuelle, de maturité, d'exposition à la complexité de la vie intérieure et surtout d'attention aux questions des relations plus qu'à celles des contenus. L'interprétation n'est jamais figée justement parce qu'elle repose sur une dynamique des relations à l'intérieur du champ symbolique de chacun. Évidemment, on peut essayer de proposer des interprétations sociales, collectives, mais il faudra toujours retenir dans tous ces cas que ce genre d'interprétation ne peut être que fondée sur des hypothèses personnelles et que ce sera l'accumulation des consensus qui montrera non pas que l'interprétation est juste mais que l'hypothèse est valable. 